\title{
Characteristics of Austenitic Stainless Steel Nitrided in a Hybrid Glow Discharge Plasma
}

\author{
R. M. Oliveira, ${ }^{*}$ M. Ueda, and L. L. G. Silva \\ Associated Plasma Laboratory, National Institute for Space Research, São José dos Campos, S. Paulo, Brazil \\ H. Reuther \\ Institute for Solid State and Materials Research, Center Rossendorf, Dresden, Germany \\ C. M. Lepienski \\ Department of Physics, Federal University of Paraná, Curitiba, Brazil
}

(Received on 17 April, 2009)

\begin{abstract}
A nitriding process based on two distinct nitrogen glow discharge modes, with sample temperatures ranging from $3800^{\circ} \mathrm{C}$ to $480{ }^{\circ} \mathrm{C}$, was employed to treat the surface of austenitic stainless steel (SS 304). The temperature is controlled exclusively by switching the operation conditions of the discharges. First mode of operation is the conventional one, named cathodic, which runs at higher pressure values $(1 \mathrm{mbar})$ in comparison to the second mode, named anodic, which runs at the pressure range of $10^{-3}-10^{-2}$ mbar. Cathodic mode is used to quickly heat the sample holder, by the high ion flux. On the other hand, in the anodic mode, due to the lower operation pressure, higher effective ion acceleration takes place, which allows deeper ion implantation into the sample surface. This hybrid process was thoroughly explored regarding the duty cycle and conditions of operation, to achieve optimal performance of the treatments, which led to the attainment of surface hardness for samples of AISI SS 304 as high as $20 \mathrm{GPa}$ and improvements including higher elastic modulus and resistance against corrosion. Detailed comparison among samples treated by this process with others treated by conventional method was done using nanoindentation, Auger Electron Spectroscopy (AES) and corrosion resistance testing.
\end{abstract}

Keywords: plasma nitriding, hybrid process, austenitic stainless steel, corrosion resistance, hardness, elastic modulus

\section{INTRODUCTION}

Plasma nitriding is usually performed at high temperatures and low voltages in a glow discharge plasma atmosphere [1]. However, the high temperature used in conventional nitriding process is not adequate to treat stainless steel (SS), due to the deleterious effect to its corrosion behavior [2,3]. Temperatures in excess of $480{ }^{\circ} \mathrm{C}$ during nitriding in SS cause precipitation of chromium nitride [4], albeit they are mandatory in this case to the achievement of enhanced mechanical and tribological properties. It is generally accepted that high corrosion resistance of SS is due to the formation of a protective oxide-hydroxide film, and that chromium oxide is responsible for the passivating behavior $[5,6]$.

Several works highlighted the desirability of performing nitriding at low temperatures [7-12] and some industrial solutions for these cases were investigated [9,13-15]. Moreover, other alternative solutions were also attempted recently. Borges et al. [10] added hydrogen/acetylene to nitrogen gas in order to decrease chromium precipitation during process. Muratore et al. [16] used an electron beam to generate the plasma and performed nitriding of SS in moderate temperature values. Furthermore, plasma immersion ion implantation (PIII) [17-20] and also a hybrid of PIII and nitriding were also used to treat stainless steels [21-22]. However, the possibility of lowering the process temperature through conventional ion nitriding has not yet been systematically explored.

One of the difficulties faced during nitriding of materials that are sensitive to temperature in conventional process is

*Electronic address: rogerio@plasma. inpe.br that the workpiece is the plasma cathode itself [9]. In this case, unpredictable heating of the workpiece over time may occur due to variations of the discharge current. It is clear that conventional nitriding is not an ideal solution to treat SS and some alternative mechanism must be used to counterbalance the undesirable effect of the high temperature.

As a sequence to our previous investigation [23-25], this paper describes additional results of a hybrid nitriding process combining two distinct discharge conditions, which led to the attainment of excellent mechanical and tribological properties for samples of austenitic SS 304, with improvement of its corrosion resistance.

\section{EXPERIMENTAL}

Ion nitriding processes were performed in a 60 liters water refrigerated stainless steel chamber. Vacuum system is composed of a two-stage rotary-pump $\left(10 \mathrm{~m}^{3} / \mathrm{h}\right)$, a mechanical booster pump based on Roots principle $\left(375 \mathrm{~m}^{3} / \mathrm{h}\right)$ and a diffusion pump. Base pressure of $2 \times 10^{-5}$ mbar is established before the treatments. Through adequate switching of the valves of the pumping system, this experiment runs in the operation pressure window varying from $10^{-3}$ mbar to 5 mbar. Argon is used previously to the treatment of the samples surface for their cleaning, by means of low pressure glow discharge. Nitrogen flux is controlled by using a needle valve. AISI 304 SS samples consisting of disks with $30 \mathrm{~mm}$ in diameter, can be fixed to the sample holder, up to 16 simultaneously. Sample temperature was monitored by a calibrated optical pyrometer. The set up used to perform this experiment is shown in figure 1.

The hybrid ion nitriding process was performed using two distinct discharge conditions. In the conventional one, 
named cathodic mode, the sample holder plays the role of the cathode of the discharge itself, being polarized to negative voltages of about $700 \mathrm{~V}$, with operation pressure of 1-2 mbar. In this case, the plasma remains concentrated around the sample holder, allowing a fast rise of the sample temperature. The other discharge mode, named anodic, is switched on with the aid of an electron source (filament) and by positively polarizing a second electrode, the anode, to $700 \mathrm{~V}$, which draws a current of $600 \mathrm{~mA}$. In this case, operation pressure is around $8 \times 10^{-3}$ mbar and the plasma spreads out to fill all of the vacuum chamber. The sample holder remains negatively polarized to attract the ions from this plasma. The tungsten filament can be switched off after the start up of this anodic discharge, but it may also be used to control the plasma potential.

Mechanical tests in the samples for the determination of hardness and elastic modulus were performed in a XP Nanoindenter (MTS Nanoinstruments). AES was carried out in a Microlab 310F model from Fisons Instruments, with a field emission electron source of $10 \mathrm{keV}$ and hemispherical sector analyzer. The potentiodynamic polarization curves were obtained by means of an EG\&G PAR 283 potenciostat, using a conventional three-electrode glass cell. AISI SS 304 steel sliced samples were used as the working electrodes. The counter electrode was a graphite rod and a calomel electrode served as a reference. Polarization curves were acquired in a $3 \% \mathrm{NaCl}$ solution, $\mathrm{pH}=6$ and recorded in electropositive direction, at a sweep rate of $0.33 \mathrm{mV} / \mathrm{s}$.

\section{RESULTS}

Plasma density, for cathodic and anodic modes, was measured by double Langmuir probe, which was placed $10 \mathrm{~cm}$ far from the sample holder in order to avoid damage to the circuitry, due to possible voltage arcs, since the cathode was polarized to high DC voltages during the measurements.

As can be seen in figure 2, the plasma density decreased for the cathodic mode from $1.1 \times 10^{9} \mathrm{~cm}^{-3}$ to $2 \times 10^{8} \mathrm{~cm}^{-3}$, for an increase of the pressure from $3 \times 10^{-1}$ to $1 \mathrm{mbar}$, respectively. Actually, with the increase of the pressure, it was verified that the plasma concentrates around the sample holder and there is a decrease of the density, measured by the double probe. This decrease of the density is a consequence of a shift of the plasma volume.

An advantage of using the cathodic mode, running in higher pressures, is that the concentration of the plasma around the sample holder causes a fast increase in the temperature of the samples, due to the high rate of flux of ions reaching them. In fact, ions are drawn from the plasma in large amount in this case, increasing the ion collision frequency, resulting in small ion mean free path (about 0.13 $\mathrm{cm})$. This mode is used initially to start up the process and whenever it is necessary to increase the sample temperature.

In the anodic discharge, plasma density ranges from ( 2 to 4) $\times 10^{9} \mathrm{~cm}^{-3}$ for the overall pressure window of operation. Ion mean free path in this case is as large as $65 \mathrm{~cm}$. When operating in the anodic mode, nitrogen ions gain large energy before hitting the samples, since they barely undergo collisions or charge transfer, resulting in higher effective ion ac- celeration which allows deeper implantation of the ions into the sample surface. It is important to stand out that the heating of the samples decreases slowly for this case and it is the main part of this hybrid treatment, running during $75 \%$ of the total treatment time.

The hybrid process was explored thoroughly regarding the duty cycle and conditions of operation. Optimal performance was obtained in a combined treatment lasting 2.5 hours, with a duty cycle of $3 / 1$ regarding the duration of the anodic discharge in relation to the cathodic one. Actually, the control of the temperature is the key point of this treatment, defining the duty cycle of the process. The sample temperature was controlled to vary in the range of 380 to $480{ }^{\circ} \mathrm{C}$. These limits were chosen to avoid chromium precipitation, but at the same time to ensure enough heating of the samples to maximize ion diffusion through the material layer.

In order to verify the separate effects of the cathodic and anodic discharges on the samples, two sets of them were submitted to each discharge condition. The first ones were treated by cathodic discharge for 1 hour, the temperature was kept controlled in the upper level of $480{ }^{0} \mathrm{C}$. The second set of samples was treated by anodic discharge for 2.5 hours in this case, the temperature remained stable in $310^{\circ} \mathrm{C}$. The characteristics of both sets of samples were then compared with the ones of the hybrid case.

We had already reported $\mathrm{x}$-ray diffraction analysis performed in samples treated by cathodic, anodic and hybrid discharges[23], which revealed the presence of martensite through the formation of $\alpha$-phase for the first case, in contrast to the formation of nitrogen rich expanded austenitic $\gamma_{N}$ phase for the anodic and hybrid discharges. In the case of the hybrid discharge, we verified the formation of $\gamma_{N}$ phase with relative intensity of the diffraction peaks greater than of primary austenitic phase, indicating that $\gamma_{N}$ layer was thick and the nitrogen abundant in the near surface layer. In this paper, detailed Auger analysis was carried out in the samples treated during 2.5 hours by anodic and hybrid discharges, as shown in figures $3 a$ and $3 b$, respectively. In figure $3 a$, it is possible to verify that nitrogen penetrates the sample up to $125 \mathrm{~nm}$ depth, being $25 \%$ the atomic percentage reached in the near surface layer. For the hybrid treatment, the peak nitrogen atomic percentage is around $30 \%$ (nearest to the surface) and the total penetration is about $1.6 \mu \mathrm{m}$ depth. From the comparison between both treatments it is clear that the low values of pressure and temperature led to the formation of a thin treated layer for the anodic case, in contrast to a much thicker layer for the hybrid process. In fact, in the anodic discharge temperature reached only $310{ }^{0} \mathrm{C}$ producing conditions for low nitrogen diffusion. This may explain the thinner thickness of the treated layer. In the hybrid treatment, the higher range of temperature used can explain the attainment of more than tenfold increase of the thickness of the treated layer, with a fairly constant nitrogen concentration.

Even though the anodic process has resulted in a shallow treated surface layer, the effectiveness of this treatment seemed to be quite satisfactory regarding the fairly hard case layer produced, with a twofold increase in hardness in comparison with the standard sample, as can be seen by the results of nanoindentation shown in figure $4 \mathrm{a}$. Furthermore, figure $4 \mathrm{a}$ also shows that the cathodic treatment performed 


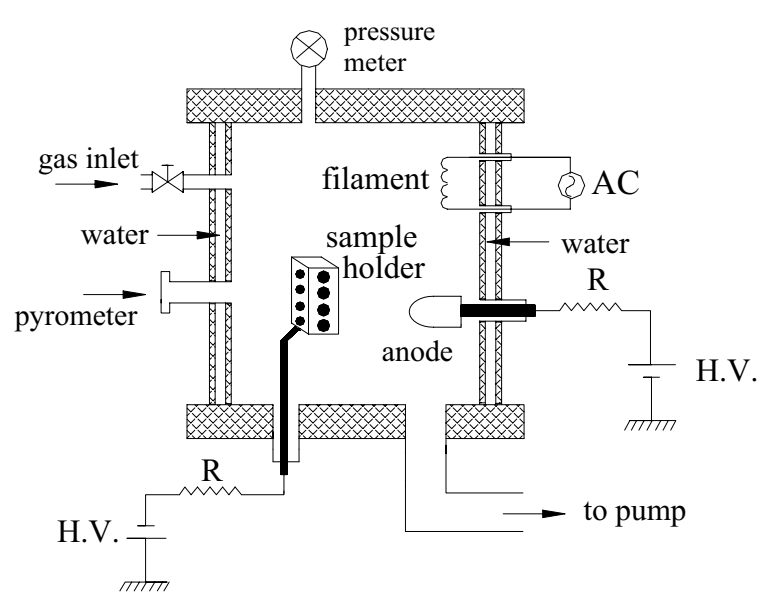

FIG. 1: Experimental setup used for hybrid ion nitriding.

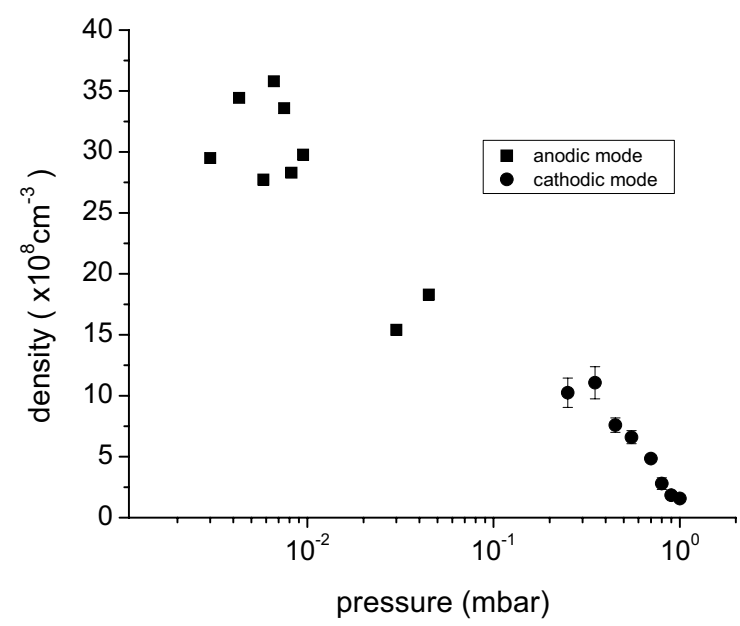

FIG. 2: Variation of the plasma density with the pressure of the discharge for cathodic and anodic processes.

in moderate and controlled temperature did not improve the hardness of the samples. On the contrary, a slight degradation occurred. In contrast, the hardness of the samples treated by the hybrid process increased almost by 4 times, compared to that of the untreated material, showing the higher resistance offered by the treated layer to plastic deformation.

The different kind of treatments (cathodic, anodic and hybrid) had a clear influence on the elastic modulus $(E)$ of the stainless steel samples, as can be seen in figure $4 \mathrm{~b}$. Largest variation of $E$ was observed for the layer treated by the hybrid method, with an increase of about $30 \%$ for the deformation depth up to approximately $100 \mathrm{~nm}$. For deeper deformations, as the indenter penetrates more profoundly in the direction to the bulk of the material, the value of $E$ begins to decrease, as expected. However, the value of $E$ remains higher than that of the standard sample for deformation until $250 \mathrm{~nm}$. The anodic treatment also caused an increase in the value of $E$ of about $10 \%$ for the deformation in $50 \mathrm{~nm}$. However, as the treated layer was thinner than the hybrid case, as shown by Auger measurements, this value of $E$ remained
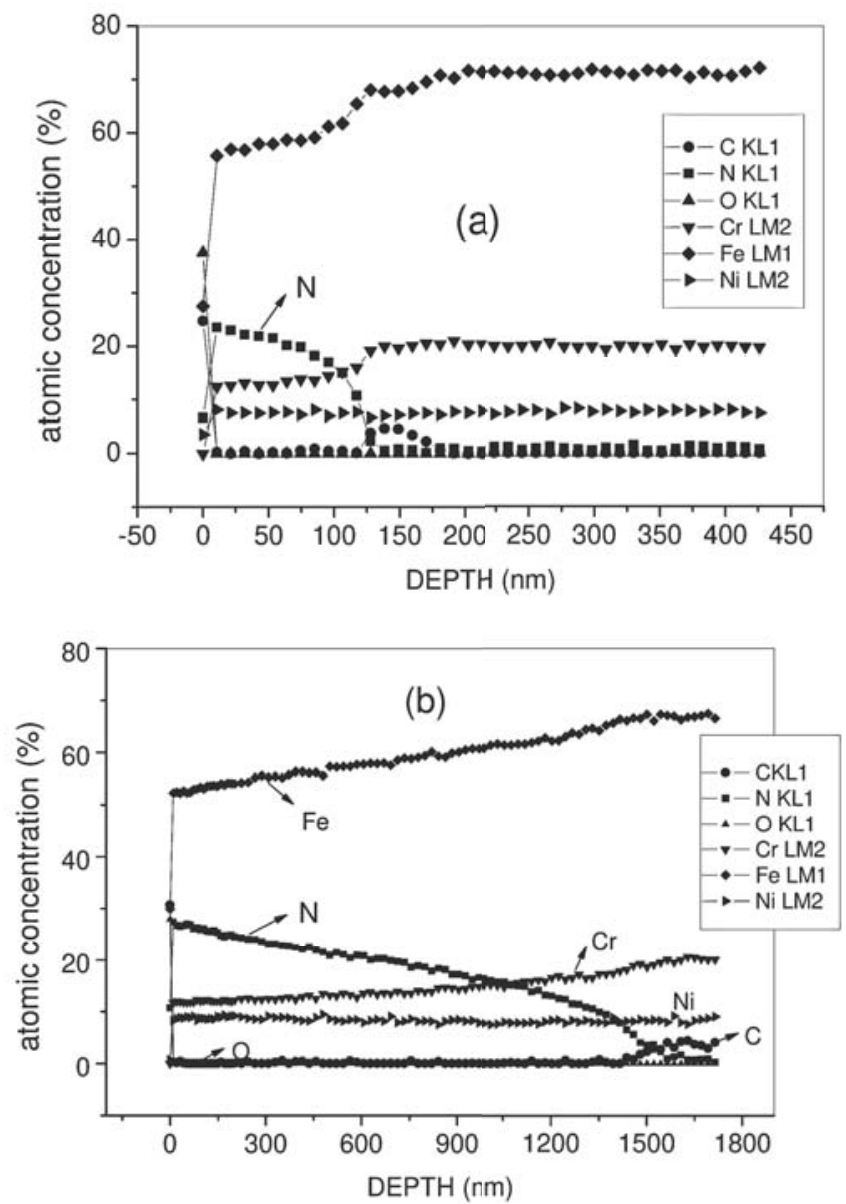

Figure 3

FIG. 3: a) Auger Electron Spectroscopy (AES) for sample treated under anodic discharge during 2.5 hours b) AES for sample treated under hybrid discharge during 2.5 hours.

higher than the untreated steel for lower deformation length (about $140 \mathrm{~nm}$ ). It should be noticed that the increased values of $E$ for the anodic case, and mainly for the hybrid treatment, indicate a good mechanical resistance for these samples. On the other hand, a decrease on the value of $E$ was observed for the samples treated by the cathodic discharge, reaching the same value of the untreated material for deformation depth of about $800 \mathrm{~nm}$.

The results of both mechanical properties, the hardness and the elastic module, once more corroborate with the effectiveness of the nitriding performed in low pressure and moderate temperature. In fact, lowering the work pressure while keeping the temperature controlled in an intermediate range, has the effect of increasing the hardness and the elasticity of the SS 304.

A corrosion test was performed, as shown in figure 5, in order to verify the corrosion behavior of the samples treated under the hybrid discharge conditions with controlled temperature, and the ones treated by conventional method, with high temperature values $\left(\sim 600^{\circ} \mathrm{C}\right)$. Corrosion degradation becomes evident for the conventional method, since corrosion current density is two orders of magnitude higher than 

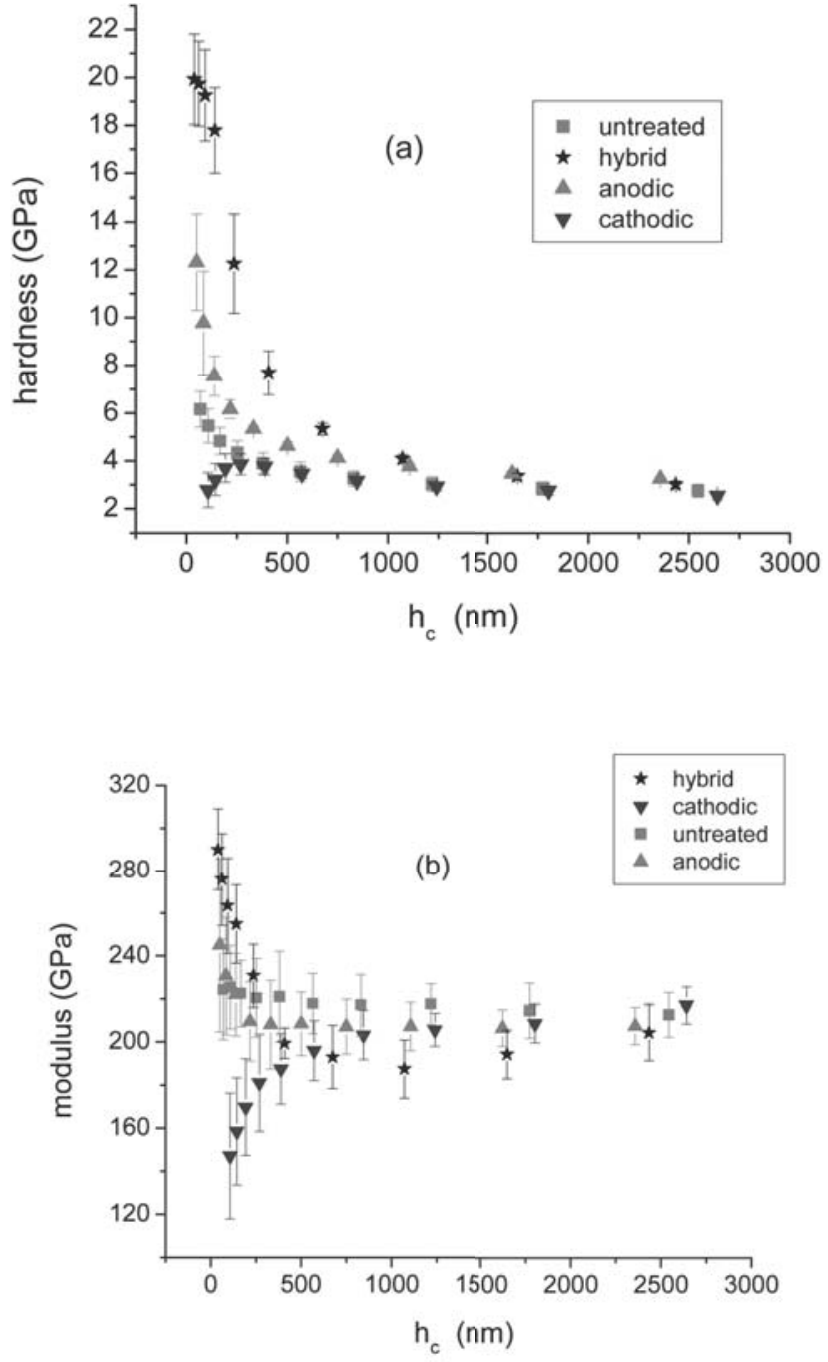

FIG. 4: Nano-indentation measurements for untreated sample in comparison with the ones treated by cathodic, anodic and hybrid discharges. a) hardness b) elastic modulus

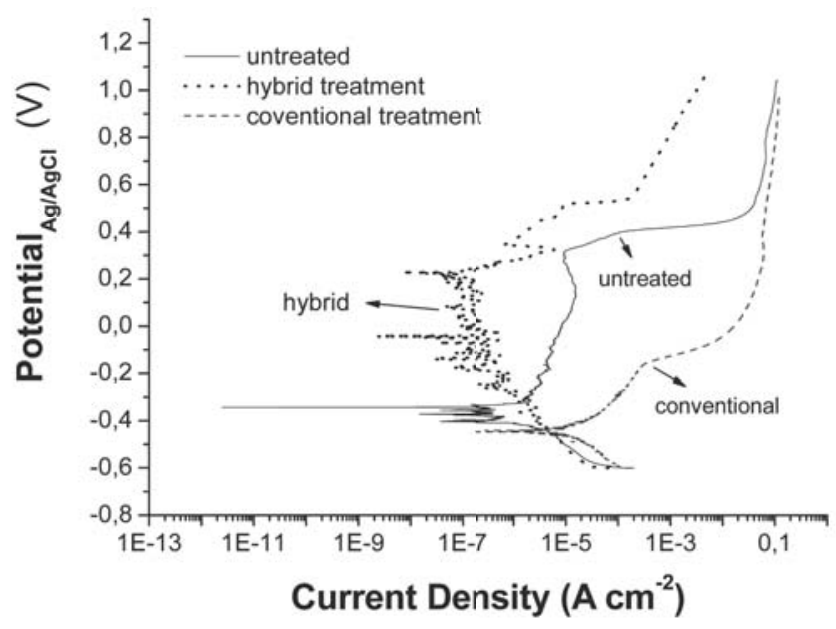

FIG. 5: Corrosion test for treated samples of SS304 (conventional nitriding at $600{ }^{\circ} \mathrm{C}$ and hybrid process), in comparison to a standard one (untreated). the one measured on the standard sample. Moreover, the corrosion potential is lower for the conventional treatment, indicating that the beginning of the corrosion starts earlier for this case. Adversely, the measurements for the hybrid case showed a shifting of the corrosion potential towards higher values, which means that the material is nobler concerning its corrosion properties. Also, the decrease of almost one order of magnitude for the current density in this case in comparison to the untreated material allow us to conclude that once the corrosion is initiated, it would occur in a slower pace for the hybrid sample.

\section{CONCLUSION}

This experiment was intended to extend the benefits of ion nitriding to lower temperatures, avoiding corrosion degradation. This is accomplished by using a hybrid treatment in which two processes running in high and low pressures were used, alternately. Sample heating was due to the ion collision with the samples surface, without using any auxiliary means. The advantage of using the low pressure was the higher effective ion acceleration achieved which allows deeper implantation of the ions into the sample surface. However, since ion bombardment does not significantly heat the workpiece under the conditions of low pressure values, the condition of the discharge was switched to higher pressures during $25 \%$ of the time to rise the sample temperature. This combined process allowed ion diffusion and efficient ion implantation of the hybrid treatment. In fact, we showed that the low pressure plasma obtained by the anodic discharge alone cannot supply sufficient amount of nitrogen to build up a high concentration of nitrogen in the surface of the samples. When the hybrid process is used, the increase of the temperature promotes large ion diffusion, compensating this negative effect.

As previously stated, temperature has a very strong influence on nitrogen diffusion, so a much thicker layer was produced in the hybrid case. Auger measurements showed a nitrogen penetration of only up to $125 \mathrm{~nm}$ for the low pressure case, against a surface layer of $1.6 \mu \mathrm{m}$ depth with fairly constant nitrogen concentration attained by the hybrid treatment. These treated layers caused a twofold and a fourfold increase in the surface hardness of SS304, besides a $10 \%$ and $30 \%$ increase in the elastic modulus, respectively.

It is worthwhile to notice that ion diffusion coefficient is possibly similar for hybrid and cathodic processes, since maximum temperature achieved in both cases are similar too. The best surface properties achieved by the first in comparison to the last just laws in the lower pressure stage of the hybrid process, which lasted up to $75 \%$ of the treatment. For this case, ions reach the workpiece with much more energy than it happens in conventional nitriding, which is performed only at high pressure values.

The addition of nitrogen to austenitic SS 304 led to the improvement of the pitting resistance. Several mechanisms had previously been proposed for this phenomenon [26]. This improvement may be attributed to the formation of an expanded austenitic layer containing chromium oxide as well as a nitrogen enrichment in the top surface. In our ex- 
periments, nitrogen played a multiple role on SS 304, by increasing pitting resistance and strength. In summary, SS 304 is hardenable by ion nitriding carried out in moderate tem- perature range and low pressure. The treated layer presents higher elastic module and improved corrosion resistance.
[1] X. B. Tian, S. C. H. Kwok, L. P. Wang and P. K. Chu. Materials Sci. and Engineering A 326 (2002), p. 348.

[2] M. Samandi, B. A. Shedden, D. I. Smith, G. A. Collins, R. Hutchings and J. Tendys. Surf. Coat. Technol. 59 (1993), p. 261.

[3] R. Valencia, R. López-Callejas, A. Muñoz-Castro, S. R. Barocio, E. Chávez A, and O. Godoy-Cabrera, Braz. J. Phys 34 (2004), p. 1594.

[4] Z.L. Zhang and T. Bell. Surf, Eng 1 (1985), p. 131.

[5] A. Hannani and F. Kermiche Transactions of the Institute of Metal Finishing 76 (1998), p. 114.

[6] P. Marcus and E. Protopopoff, J. Electrochem. Soc. 137 (1990), p. 2709.

[7] E. Menthe and K.-T.Rie, Surf. Coat. Technol. 116-119 (1999), p. 199.

[8] N. Renevier, P. Collignon, H. Michel and T. Czerwiec, Surf. Coat. Technol. 111 (1999), p. 128.

[9] T. Czerwiec, N. Renevier and H. Michel, Surf. Coat. Technol. 131 (2000), p. 267

[10] C. F. M. Borges, S. Hennecke and E. Pfender, Surf. Coat. Technol. 123 (2000), p.112.

[11] M. P. Fewell, J. M. Priest, M. J. Baldwin, G. A. Collins and K. T. Short, Surf. Coat. Technol. 131 (2000), p. 284.

[12] S. K. Kim, J. S. Yoo, J. M. Priest and M. P. Fewell, Surf. Coat. Technol. 163 (2003), p. 380

[13] M. K. Lei and Z. L. Zhang, J. Vac. Sci. Technol., A.Vac. Surf, Films 15 (1997), p. 421.

[14] M. J. Baldwin, M. P. Fewell, S.C. Haydon, S. Kumar, G.A.Collins, K.T. Short and J. Tendys. Surf. Coat. Technol.
98 (1998), p. 1187.

[15] J.M. Priest, M.J. Baldwin, M.P. Fewell, S.C. Haydon, G.A. Collins, K.T. Short and J. Tendys, Thin Solid Films 345 (1999), p. 113.

[16] C. Muratore, D. Leonhardt, S. G. Walton, D. D. Blackwell, R. F. Fernsler and R. A. Meger. Surf. Coat. Technol. 191 (2005), p. 255.

[17] P.K. Chu, X. Lu, S.S.K. Iyer, N.W. Cheung, Solid State Technol. 40 (1997) S9.

[18] B. Tian, Z. M. Zeng, T. Zhang, B.Y. Tang, P. K. Chu, Thin Solid Films 366 (2000), p.150.

[19] X. M. Zhu, M. K. Lei, Surf. Coat. Technol. 131 (2000), p. 400.

[20] K. Ram Mohan Rao, S. Mukherjee, S.K. Roy, E. Richter, W. Möller, I. Manna, Surf. Coat. Technol. 201 (2007), p. 4919.

[21] X. B. Tian, Z. M. Zeng, B. Y. Tang and P. K. Chu in: 27th Int. Conf. on Metallurgical Coatings and Thin Films (ICMCTF), San Diego, CA (2000), p. 1999.

[22] M. Ueda, G. F. Gomes, K. G. Kostov, H. Reuther, C. M. Lepienski, P. C. Soares Jr., O. Takai and M. M. Silva, Braz. J. Phys 34 (2004), p. 1632.

[23] R. M. Oliveira, M. Ueda, I. H. Tan, L. Hoshida, C. B. Mello, Plasma Processes and Polymers 4, S1 (2007), p. 655.

[24] M. Ueda, G. F. Gomes, Nucl. Instr. and Meth. in Phys. Res. B 206 (2003) p.749.

[25] G. F. Gomes and M. Ueda, J. Appl. Phys., 94 (??) (2003) p. 5379.

[26] R. Sabot, R. Devaux, A.M. De Becdelievre and C. DuretThual, Corrosion Sci. 33 (1992), p. 1121. 